\title{
Desempenho de métodos de estimativa da evapotranspiração de referência para a região da Serra da Mantiqueira, MG
}

\author{
Performance of the reference evapotranspiration estimating methods for the Mantiqueira range region, \\ MG, Brazil
}

\author{
Donizete dos Reis Pereira ${ }^{\mathrm{I}}$ Sílvia de Nazaré Monteiro Yanagi ${ }^{\mathrm{I}}$ Carlos Rogério de Mello ${ }^{\mathrm{II}}$ \\ Antônio Marciano da Silva' Lucas Alves da Silva ${ }^{I}$
}

\section{RESUMO}

Objetivou-se, com o presente trabalho, avaliar o desempenho de métodos de estimativa da evapotranspiração de referência (ETo) nas escalas diária e mensal, durante os períodos chuvoso (outubro a março) e seco (abril a setembro), nos anos de 2007 e 2008, para a bacia hidrográfica do ribeirão Lavrinha, região da Serra da Mantiqueira, Minas Gerais (MG). O método de Penman-Monteith-FAO foi utilizado como referência. Para avaliação do desempenho dos métodos, foram considerados os coeficientes de correlação $(r)$ e determinação $\left(r^{2}\right)$, concordância de Willmott (d), desempenho (c) de Camargo \& Sentelhas e erro padrão de estimativa (EPE). Em geral, verificou-se que os métodos que envolvem radiação solar foram mais precisos na estimativa da ETo em relação aos métodos que envolvem apenas a temperatura do ar. Foram obtidas melhores estimativas pelos métodos de Jensen-Haise, FAO Radiação, Penman e Blaney-Criddle FAO para ambos os períodos, com coeficientes de desempenho entre "ótimo” $e$ "muito bom". Os métodos Jensen-Haise, FAO Radiação e Blaney-Criddle FAO são mais indicados para estimativas em escala diária, para a região da Serra da Mantiqueira, por apresentarem maior precisão e formulações mais simples, enquanto que o método de Jensen-Haise apresentou o menor EPE nas estimativas em escala mensal.

Palavras-chave: evapotranspiração, métodos de estimativa, Serra da Mantiqueira, balanço hídrico.

\section{ABSTRACT}

The objective of this research was to evaluate the performance of reference evapotranspiration (ETo) estimating methods, in daily and monthly intervals, considering the rainysummer (October to March) and dry-winter (April to September) seasons, in 2007 and 2008, in Mantiqueira range region, MG. To evaluate the performance methods were considered: the correlation ( $r$ ) and determination $\left(r^{2}\right)$ coefficients, Willmott agreement (d), e Camargo \& Sentelhas performance (c) and standard error estimate (SEE), taking Penman-Monteith-FAO as reference. It was verified that the methods based on solar radiation were more accurate than those based only on air temperature. Jensen-Haise, Radiation FAO, Penman and Blaney-Criddle FAO have produced better estimates in both seasons, obtaining good reliability on ETo estimate for the region, presenting "high" and "very good" performance. Jensen-Haise, Radiation FAO and Blaney-Criddle FAO are the most indicated methods in daily scale to the Mantiqueira range region due to present simpler mathematical structure and good performance, while the Jensen-Haise method presented the smallest standard error for the monthly scale.

Key words: evapotranspiration, estimating methods, Mantiqueira Range, water balance.

\section{INTRODUÇÃO}

O processo simultâneo de perda de água para a atmosfera por meio da evaporação do solo e transpiração das plantas é denominado evapotranspiração (ET), sendo um elemento climatológico fundamental que corresponde ao processo oposto à chuva (PEREIRA et al., 1997; CAMARGO \& CAMARGO, 2000). AET é controlada pelo balanço de energia, pela demanda atmosférica, pelo suprimento de água do solo às plantas e pelas características fisiológicas das plantas (PEREIRA et al., 1997; MENDONÇA et al., 2003). No entanto, para evitar conflitos, foi introduzido o conceito de evapotranspiração de referência (ETo), cuja definição

IPrograma de Pós-graduação em Engenharia Agrícola (PPGEA), Departamento de Engenharia, Universidade Federal de Lavras (UFLA), Lavras, MG, Brasil.


correspondência. 
está associada ao processo de perda de água para a atmosfera considerando uma superfície extensa, coberta por grama com altura entre 0,08 e $0,15 \mathrm{~m}$, em crescimento ativo, cobrindo totalmente o solo e sem deficiência de água (DOORENBOS \& PRUITT, 1977).

A determinação da ETo pode ser feita por meio de métodos de estimativa (indiretos), os quais são menos onerosos que os diretos, uma vez que sua aplicação é baseada em elementos meteorológicos medidos em estações apropriadas (PEREIRA et al., 1997). Dentre os modelos indiretos, a Organização das Nações Unidas para Alimentação e Agricultura (FAO) recomenda o uso do método de Penman-Monteith como método padrão para a estimativa de ETo, devendo ser utilizado na avaliação de outros métodos de estimativa (ALLEN et al., 1998), uma vez que este mostra-se eficiente em diversas condições climáticas. Entretanto, esse método necessita de grande número de elementos meteorológicos, que nem sempre estão disponíveis em algumas regiões, levando, dessa forma, ao uso de equações mais simples (CAMARGO \& CAMARGO, 2000).

Antes de aplicar um método para determinado local, é necessário verificar o desempenho deste e, quando necessário, fazer calibrações a fim de minimizar erros de estimativa. Esse desempenho tem sido analisado com a comparação dos respectivos métodos ao método de Penman-Monteith-FAO. Assim, alguns trabalhos foram desenvolvidos nessa linha, para diferentes regiões do Brasil, destacando-se os de CONCEIÇÃO \& MANDELLI(2005), OLIVEIRA et al. (2005) e BORGES \& MENDIONDO (2007). No âmbito internacional, recentemente foram calibrados e comparados alguns modelos de estimativa de ETo ao de Penman-Monteith, para o Tibet (China) por YE et al. (2009). CHAUHAN \& SHRIVASTAVA(2009) também compararam o desempenho de métodos indiretos em relação ao de Penman-Monteith para a região do reservatório Mahanadi, na Índia, e chegaram a correlações significativas e importantes para aplicação desses métodos em condições menos favoráveis em termos de disponibilidade de dados. Observa-se, portanto, que a análise estatística do desempenho de métodos mais simples em relação ao método de PenmanMonteith-FAO consiste de um estudo relevante para aplicação mais precisa ao manejo de irrigação, à simulação hidrológica e à análise climática de uma determinada região.

O objetivo deste trabalho foi avaliar o desempenho de métodos indiretos de estimativa da evapotranspiração de referência em comparação com o método padrão Penman-Monteith-FAO durante o período seco e chuvoso, em escalas diária e mensal, para as condições climáticas da região da Serra da Mantiqueira, MG.

\section{MATERIAL E MÉTODOS}

A área de estudo corresponde à bacia hidrográfica do ribeirão Lavrinha, na região da Serra da Mantiqueira, sendo afluente direto do Rio Grande e representativa em termos hidrológicos e climáticos (ÁVILA, 2008). Está localizada entre as coordenadas UTM “7551369" - “7554369” de latitude S e "553842” “557742” de longitude W e altitudes entre 1144 e 1739m.

A região possui clima $C w b$ pela classificação de Köppen, com invernos frios e menos úmidos que os verões, que são amenos e mais chuvosos. A precipitação média anual é de $2150 \mathrm{~mm}$, e as temperaturas médias no mês mais quente e no mês mais frio são, respectivamente, de $19^{\circ} \mathrm{C}$ e $11^{\circ} \mathrm{C}$. Para a estimativa da ETo, foram monitorados, por meio de uma estação meteorológica automática completa (Campbell Scientific, INC, modelo WeatherHawk), os elementos climáticos temperatura do ar, temperatura do ponto de orvalho, umidade relativa do ar, velocidade e direção do vento, radiação solar global, precipitação, pressão barométrica e densidade do ar durante os anos de 2007 e 2008, a cada 30 minutos. Os valores diários desses elementos meteorológicos, com exceção da precipitação, a qual foi obtida pela soma dos valores de cada intervalo, foram gerados a partir da média dos valores de 30 minutos (média de 48 valores). É importante relatar que não existe nenhuma estação meteorológica do INMET na região da Serra da Mantiqueira, ou seja, não há disponibilidade de séries históricas. Portanto, os dados disponíveis para este estudo foram gerados a partir da implantação do monitoramento hidroclimático na referida bacia hidrográfica, fazendo parte de um projeto de Pesquisa e Desenvolvimento em parceria com a CEMIG/ANEEL.

$O$ cálculo da ETo pelo método de PenmanMonteith (EToPM), padronizado pela FAO, seguiu-se a metodologia descrita por ALLEN et al. (1998) do seguinte modo:

$$
\text { EToPM }=\frac{0,408 \cdot \Delta \cdot\left(\mathrm{R}_{\mathrm{n}}-\mathrm{G}\right)+\gamma \cdot \frac{900}{\mathrm{~T}+273} \cdot \mathrm{u}_{2} \cdot\left(\mathrm{e}_{\mathrm{s}}-\mathrm{e}_{\mathrm{a}}\right)}{\Delta+\gamma \cdot\left(1+0.34 \cdot \mathrm{u}_{2}\right)}
$$

em que EToPM é a evapotranspiração de referência $\left(\mathrm{mm} \mathrm{d}^{-1}\right) ; \Delta$ é a declividade da curva de pressão de saturação do vapor $\left(\mathrm{kPa}^{\circ} \mathrm{C}^{-1}\right)$; Rn é o saldo de radiação (MJ m-2 d-1); G é o fluxo de calor no solo $\left(\mathrm{MJ} \mathrm{m}^{-2} \mathrm{~d}^{-1}\right)$, na escala diária; $\mathrm{G}=0 ; \gamma$ é o coeficiente psicrométrico $\left(\mathrm{kPa}^{\circ} \mathrm{C}^{-1}\right) ; \mathrm{u}_{2}$ é a velocidade do vento a $2 \mathrm{~m}$ de altura $\left(\mathrm{m} \mathrm{s}^{-1}\right)$; $\mathrm{e}_{\mathrm{s}}$ é a pressão de saturação do vapor d'água $(\mathrm{kPa})$; e é a pressão atual do vapor d'água $(\mathrm{kPa})$; e $\mathrm{T}$ é a temperatura média diária do $\operatorname{ar}\left({ }^{\circ} \mathrm{C}\right)$. 
Os demais métodos foram aplicados de acordo com PEREIRA et al. (1997):

a)Makkink (EToM): EtoM =0,61 $\mathrm{W} \mathrm{R}_{\mathrm{s}}-0,12 \quad$ (2), em que ETo é a evapotranspiração de referência ( $\mathrm{mm}$ $\operatorname{dia}^{-1}$ ); W é um fator de ponderação, sendo estimado por meio das expressões lineares propostas por WILSON \& ROUSE (1972) e VISWANADHAM et al. (1991) para temperaturas de bulbos úmidos variando de 0 a $16^{\circ} \mathrm{C}$ e 16,1 a $32^{\circ} \mathrm{C}$, respectivamente.

b)FAO Radiação Solar(EToR): EtoR $-c_{o}+c_{1}$.W.R $R_{s}$ (3), em que EToR é a evapotranspiração de referência ( $\mathrm{mm}$ $\left.\mathrm{dia}^{-1}\right), \mathrm{c}_{0}=0,30$ e $\mathrm{c}_{1}$ depende da velocidade do vento a $2 \mathrm{~m}$ de altura e da umidade relativa média do ar.

c) Jensen-Haise (EToJH): EtoJH=Rs. $(0,0252 \mathrm{~T}+0,078)$ (4), d) Linacre $(\mathrm{EToL})$ :

$$
\text { EToL }=\frac{\frac{(500 \cdot(\mathrm{T}+0,006 \cdot \mathrm{h}))}{(100-\varphi)}+15 \cdot\left(\mathrm{T}-\mathrm{T}_{\mathrm{O}}\right)}{(80-\mathrm{T})}
$$

em que EToL é a evapotranspiração de referência ( $\mathrm{mm}$ $\left.\mathrm{dia}^{-1}\right) ; \mathrm{T}_{\mathrm{o}}$, a temperatura do ponto de orvalho $\left({ }^{\circ} \mathrm{C}\right) ; \mathrm{h}$, a altitude local (m); e f, a latitude (graus).

e) Hargreaves-Samani (EToHS): EToHS =0,0023. Qo . (T0,5 . $(\mathrm{T}+17,8)$

f) Blaney-Criddle FAO (EToBC): EtoBC $=a+b \cdot p$. $(0,46 . T+8,13)$

em que EToBC é a evapotranspiração de referência ( $\mathrm{mm}$ $\mathrm{dia}^{-1}$ ); a e b são coeficientes dependentes da umidade relativa mínima mensal (\%) e da razão entre o número de horas de brilho solar na superfície da Terra e o número máximo de brilho solar no topo da atmosfera. g) Priestley-Taylor (EToPT): EToPT $=1,26 \mathrm{~W}\left(\mathrm{R}_{\mathrm{n}}-\mathrm{G}\right)(8)$, em que EToPT é a evapotranspiração de referência ( $\mathrm{mm}$ $\mathrm{dia}^{-1}$ ) e G, o fluxo de calor no solo (igual a 0 para escala diária).

h) Penman (EToP): $\mathrm{EToP}=\frac{\mathrm{W}}{\lambda} \cdot(\mathrm{Rn}-\mathrm{G})+(1-\mathrm{W}) \cdot \mathrm{f}(u) \cdot\left(e_{s}-e_{a}\right)(9)$, em que ETo é a evapotranspiração de referência (mm $\left.\operatorname{dia}^{-1}\right) ; \lambda$ é o calor latente de vaporização d'água $(2,45 \mathrm{MJ}$ $\left.\mathrm{kg}^{-1}{ }^{\circ} \mathrm{C}^{-1}\right)$ e $\mathrm{f}(\mathrm{u})$ é a função empírica do vento, que representa o termo aerodinâmico do método.

Os métodos de Hamon e Karrufa foram aplicados de acordo com metodologia proposta por BORGES \& MENDIONDO (2007), cujas equações são:

i) Hamon: $\mathrm{EToHM}=0,69 \cdot\left(\frac{\mathrm{N}}{12}\right)^{2} \cdot\left(\frac{4,95 \cdot \mathrm{e}^{0,062 \cdot \mathrm{T}}}{100}\right) \cdot 25.4$

j) Karrufa: EtoKM=0,34.p. $T^{\mathrm{k}}(11)$, em que EToKM é a evapotranspiração de referência $\left(\mathrm{mm} \mathrm{dia}{ }^{-1}\right)$; p é a porcentagem de insolação máxima diária; e k é a constante de BORGES \& MENDIONDO (2007), sendo igual a 1,2 (de agosto-março); 1,15 (abril-maio) e 1,1 (junho-julho).

A análise dos resultados foi feita pela correlação entre os valores estimados pelo método padrão e os valores estimados pelos demais modelos, baseando-se nos seguintes indicadores estatísticos: erro padrão de estimativa (EPE); coeficiente linear (a); coeficiente angular (b); coeficiente de determinação $\left(\mathrm{r}^{2}\right)$; coeficiente de correlação $(\mathrm{r})$; e no índice de concordância (d) de Willmott proposto por WILLMOTT et al. (1985) e no coeficiente de confiança ou desempenho (c) proposto por CAMARGO \& SENTELHAS (1997). Ressalta-se que a classificação do desempenho baseou-se no indicador estatístico c, e os demais indicadores servem apenas para dar suporte à classificação.

O erro padrão de estimativa (EPE), descrito por ALLEN et al. (1986), foi calculado pela equação 12:

$$
\mathrm{EPE}=\sqrt{\frac{\sum\left(\mathrm{O}_{\mathrm{i}}-\mathrm{P}_{\mathrm{i}}\right)^{2}}{\mathrm{n}-1}}
$$

em que n é o número de observações.

O índice de Willmott (d), proposto por WILLMOTT et al (1985), foi determinado pela equação 13:

$$
\mathrm{d}=1-\left[\frac{\sum(\mathrm{Pi}-\mathrm{Oi})^{2}}{\sum(|\mathrm{Pi}-\mathrm{O}|+|\mathrm{Oi}-\mathrm{O}|)^{2}}\right]
$$

em que Pi corresponde aos valores estimados pelos métodos; $\mathrm{O}_{i}$, aos valores estimados pelo método padrão Penman-Monteith; $\mathrm{O}$ é a média dos estimados pelo método padrão Penman-Monteith. Os valores de "d" podem variar de 0 , para nenhuma concordância, a 1 , para uma concordância perfeita.

O coeficiente c, proposto por CAMARGO \& SENTELHAS (1997), é obtido pelo produto entre o coeficiente de correlação (r) e o índice de Willmott (d) e interpretado de acordo com os referidos autores como: "ótimo" (c > 0,85); "muito bom" (c entre 0,76 e 0,85); "bom" (c entre 0,66 e 0,75); "mediano" (c entre $0,61 \mathrm{e}$ 0,65 ), "sofrível" (c entre 0,51 e 0,60), "mau" (c entre $0,41$ e 0,50$)$ e "péssimo" $(c<0,40)$.

\section{RESULTADOS E DISCUSSÃO}

Com relação ao ajuste dos dados estimados aos observados pelo método padrão (EToPM) (Tabela 1), ao se analisarem os indicadores estatísticos testados verifica-se que, para o período seco, o modelo EToP apresentou o melhor ajuste, tendo sido o único classificado como ótimo pelo coeficiente de confiança ou desempenho (c) e apresentado os maiores valores de $\mathrm{r}$ e $\mathrm{r}^{2}$, além de apresentar valores menores de EPE e do índice de concordância (d). Apenas o modelo EToJH foi superior em termos do EPE e do índice de concordância. Os modelos EToJH, EToR e EToBC apresentaram desempenhos ligeiramente inferiores ao modelo EToP. Os dois primeiros são aproximadamente equivalentes e o último apresentou desempenho 
Tabela 1 - Evapotranspiração média diária \pm desvio padrão $\left(\mathrm{mm} \mathrm{dia}^{-1}\right)$, erro padrão de estimativa $\left(\right.$ EPE, mm dia $\left.{ }^{-1}\right)$, coeficiente linear $(\mathrm{a})$, coeficiente angular (b), coeficiente de determinação $\left(\mathrm{r}^{2}\right)$, coeficiente de correlação $(\mathrm{r})$, coeficiente de concordância (d), coeficiente de confiança e desempenho (c) e classificação do desempenho dos modelos para os períodos chuvoso e seco na região da Serra da Mantiqueira, MG.

\begin{tabular}{|c|c|c|c|c|c|c|c|c|c|c|}
\hline Método analisado & $\begin{array}{c}\text { Período } \\
\text { analisado }\end{array}$ & ETo & EPE & $\mathrm{a}$ & $\mathrm{b}$ & $r^{2}$ & $\mathrm{r}$ & d & $\mathrm{C}$ & Classificação ${ }^{* * *}$ \\
\hline \multirow{2}{*}{ EToM } & Seco & $1,77 \pm 0,73$ & 1,03 & $0,471 * *$ & $1,059 * *$ & 0,75 & 0,87 & 0,82 & 0,71 & Bom \\
\hline & Chuvoso & $2,33 \pm 1,05$ & 0,99 & $0,620 * *$ & $1,112 * *$ & 0,92 & 0,96 & 0,85 & 0,81 & Muito bom \\
\hline \multirow{2}{*}{ EToR } & Seco & $2,61 \pm 1,23$ & 0,88 & $0,632 * *$ & $0,657 * *$ & 0,82 & 0,91 & 0,91 & 0,83 & Muito bom \\
\hline & Chuvoso & $3,49 \pm 1,77$ & 0,72 & $0,894 * *$ & $0,664 * *$ & 0,94 & 0,97 & 0,94 & 0,91 & Ótimo \\
\hline \multirow{2}{*}{ ЕTоJH } & Seco & $2,31 \pm 0,99$ & 0,64 & $0,496^{* *}$ & $0,801 * *$ & 0,79 & 0,89 & 0,94 & 0,83 & Muito bom \\
\hline & Chuvoso & $3,42 \pm 1,57$ & 0,57 & $0,669 * *$ & $0,743 * *$ & 0,92 & 0,96 & 0,96 & 0,92 & Ótimo \\
\hline \multirow{2}{*}{ EToL } & Seco & $5,08 \pm 1,40$ & 4,04 & $-0,349 * *$ & $0,531 * *$ & 0,69 & 0,83 & 0,43 & 0,36 & Péssimo \\
\hline & Chuvoso & $5,72 \pm 1,44$ & 2,60 & $-0,857 * *$ & $0,710 * *$ & 0,71 & 0,84 & 0,53 & 0,45 & Mau \\
\hline \multirow{2}{*}{ EToHM } & Seco & $2,02 \pm 0,41$ & 1,16 & - & $1,166^{* *}$ & 0,30 & 0,55 & 0,60 & 0,33 & Péssimo \\
\hline & Chuvoso & $3,31 \pm 0,46$ & 1,01 & $-2,173 * *$ & $1,628 * *$ & 0,38 & 0,62 & 0,61 & 0,38 & Péssimo \\
\hline \multirow{2}{*}{ EToKM } & Seco & $2,10 \pm 0,54$ & 1,09 & $0,317 * *$ & $0,966^{* *}$ & 0,34 & 0,58 & 0,69 & 0,40 & Péssimo \\
\hline & Chuvoso & $3,46 \pm 0,44$ & 1,00 & $-3,416^{* *}$ & $1,913 * *$ & 0,48 & 0,69 & 0,63 & 0,44 & Mau \\
\hline \multirow{2}{*}{ EToHS } & Seco & $2,89 \pm 0,78$ & 1,14 & $-0,131 * *$ & $0,859 * *$ & 0,56 & 0,75 & 0,78 & 0,58 & Sofrivel \\
\hline & Chuvoso & $4,34 \pm 0,94$ & 1,33 & $-1,347 * *$ & $1,050 * *$ & 0,67 & 0,82 & 0,71 & 0,58 & Sofrível \\
\hline \multirow{2}{*}{ EToBC } & Seco & $2,84 \pm 1,03$ & 0,95 & $0,127 * *$ & $0,782 * *$ & 0,81 & 0,90 & 0,88 & 0,80 & Muito bom \\
\hline & Chuvoso & $3,53 \pm 1,31$ & 0,53 & $0,118 * *$ & $0,875^{* *}$ & 0,89 & 0,94 & 0,95 & 0,90 & Ótimo \\
\hline \multirow{2}{*}{ EToPT } & Seco & $1,18 \pm 0,54$ & 2,01 & $1,436^{* *}$ & $0,774 * *$ & 0,22 & 0,47 & 0,52 & 0,24 & Péssimo \\
\hline & Chuvoso & $2,46 \pm 0,78$ & 0,99 & $-0,143^{* *}$ & $1,364 * *$ & 0,77 & 0,88 & 0,79 & 0,70 & Bom \\
\hline \multirow{2}{*}{ EToP } & Seco & $2,93 \pm 1,01$ & 0,87 & $-0,224 * *$ & $0,877 * *$ & 0,98 & 0,99 & 0,91 & 0,90 & Ótimo \\
\hline & Chuvoso & $3,96 \pm 1,43$ & 0,82 & $-0,129 * *$ & $0,844 * *$ & 0,98 & 0,99 & 0,92 & 0,91 & Ótimo \\
\hline
\end{tabular}

* Significativo a nível de $5 \%$ de probabilidade pelo teste $\mathrm{t}$; * Significativo a nível de $1 \%$ de probabilidade pelo teste t; NS: não significativo a nível de $5 \%$ de probabilidade pelo teste t. ${ }^{* * *}$ Classificação do desempenho, conforme Índice c, de acordo com CAMARGO \& SENTELHAS (1997).

ligeiramente inferior. Os demais modelos mostraram-se inadequados para serem aplicados para o período seco na região da Serra da Mantiqueira, visto que apresentaram grande dispersão em relação ao método de Pennan-Monteith-FAO.

Análise similar realizada para o período chuvoso permitiu classificar quatro modelos como sendo os melhores para serem aplicados na região da Serra da Mantiqueira. Em ordem decrescente, esses modelos são: EToJH, EToR, EToP e ETBC (Tabela 1). Ressalta-se que os modelos citados foram classificados como ótimo pelo coeficiente de confiança ou desempenho (c), e os modelos EToM e EToPT foram classificados como muito bom e bom, respectivamente, indicando desempenho intermediário. O uso dos demais modelos durante o período chuvoso, na região da Serra da Mantiqueira, é desaconselhável, tendo em vista os baixos desempenhos observados por meio dos indicadores estatísticos.

Apesar de a ordem de classificação do desempenho dos modelos testados não ser a mesma para os períodos seco e chuvoso, verifica-se que os modelos que apresentaram os melhores ajustes são os mesmos. Ao se analisarem os coeficientes lineares (a) e angulares (b) dos modelos empíricos ajustados (Tabela 1), observa-se que todos foram significativos $(\mathrm{P}<0,01$, teste $\mathrm{t})$, exceto o coeficiente linear do método EToHM $(\mathrm{P}>0,05$, teste $\mathrm{t})$. Deve-se salientar que esse último resultado citado seria o ideal se os demais indicadores estatísticos tivessem assumidos valores apropriados. Assim, os modelos EToP e EToBC apresentaram as melhores combinações de valores para a e b, simultaneamente aos demais indicadores estatísticos, para serem aplicados à região da Serra da Mantiqueira independentemente da estação do ano.

Entre os métodos avaliados, o método EToPT apresentou redução elevada nos indicadores estatísticos, no período seco em relação aos obtidos para o período chuvoso, mostrando a sensibilidade desse método às mudanças nas condições climáticas, pois este depende fundamentalmente da radiação solar, a qual sofre redução de acordo com a estação de verão (período chuvoso) para a de inverno (período seco) na região da Serra da Mantiqueira. Nessa mesma linha de abordagem, uma explicação complementar para esse comportamento pode ser atribuída à partição da energia 
disponível na superfície. Se esta estiver umedecida, a maior parte da energia é convertida em calor latente de vaporização, condição observada na estação chuvosa. Se, por outro lado, a superfície apresentar restrição hídrica, condição inerente à estação seca, a maior parte da energia será usada para aquecimento do ar.

CONCEIÇÃO \& MANDELLI (2005) avaliaram a adequabilidade de métodos que empregam a temperatura do ar e/ou radiação solar como variáveis de entrada comparando-os aos valores diários estimados pelo método padrão-FAO para a região de Bento Gonçalves, Rio Grande do Sul (RS), e observaram que os melhores resultados obtidos foram com os métodos que empregam a radiação global incidente como variável.

De acordo com OLIVEIRA et al. (2005), em estudo realizado na região de Goiânia, Goiás (GO), a melhor estimativa da evapotranspiração para o período seco (abril a setembro) foi obtida pelo método de Penman-FAO em comparação ao padrão-FAO, enquanto que, para o período chuvoso (outubro a março), os resultados obtidos pelo modelo de BlaneyCriddle foram os que mais se aproximaram do modelo padrão, resultados similares aos encontrados neste trabalho.

REIS et al. (2007), avaliando métodos de estimativa da ETo para Venda Nova do Imigrante, Espírito Santo (ES), em comparação ao método padrãoFAO, no período seco (junho a agosto), em escala diária, obtiveram desempenho classificado como "bom" pelos métodos EToP, EToR, EToBC, EToPT e EToM e desempenho "péssimo" pelo método de EToHS. Esses resultados foram próximos aos obtidos para o período seco do presente trabalho (Tabela 1), com exceção do método EToPT, que apresentou desempenho "péssimo" e alto EPE (2,01 mm dia $\left.{ }^{-1}\right)$.

A elevada variabilidade observada no desempenho da equação de EToPT para o período chuvoso e seco, na região da Serra da Mantiqueira (Tabela 1), pode ser explicada pelo efeito da sazonalidade das chuvas no local. Segundo PEREIRA et al. (1997), o método EToPT pode ser interpretado como uma simplificação do método EToP, no qual se retém apenas o termo associado à radiação solar corrigido por um coeficiente empírico a. SENTELHAS et al. (2000) avaliaram o efeito da sazonalidade das chuvas sobre a nas condições climáticas de Piracicaba, São Paulo (SP), e concluíram que a varia de acordo com as condições de umidade regional. Neste trabalho, foi verificado, para o período úmido, valor de a significativamente inferior ao proposto por Priestley \& Taylor e para o período seco, valor próximo ao original. Isso mostra que, para melhor uso desse método, é conveniente a adoção distinta de valores de a.

A figura 1 ilustra o comportamento médio mensal dos modelos de ETo comparados ao padrãoFAO (EToPM) para Serra da Mantiqueira, MG, em que pode-se observar um comportamento sazonal semelhante entre os modelos avaliados e o padrãoFAO. Verifica-se, ainda, que o método de EToJH foi o que melhor se ajustou aos dados de EToPM, com EPE de $0,23 \mathrm{~mm} \mathrm{dia}^{-1}$ durante o ano e altos índices de concordância $(d)$, próximos à unidade $(0,96)$,

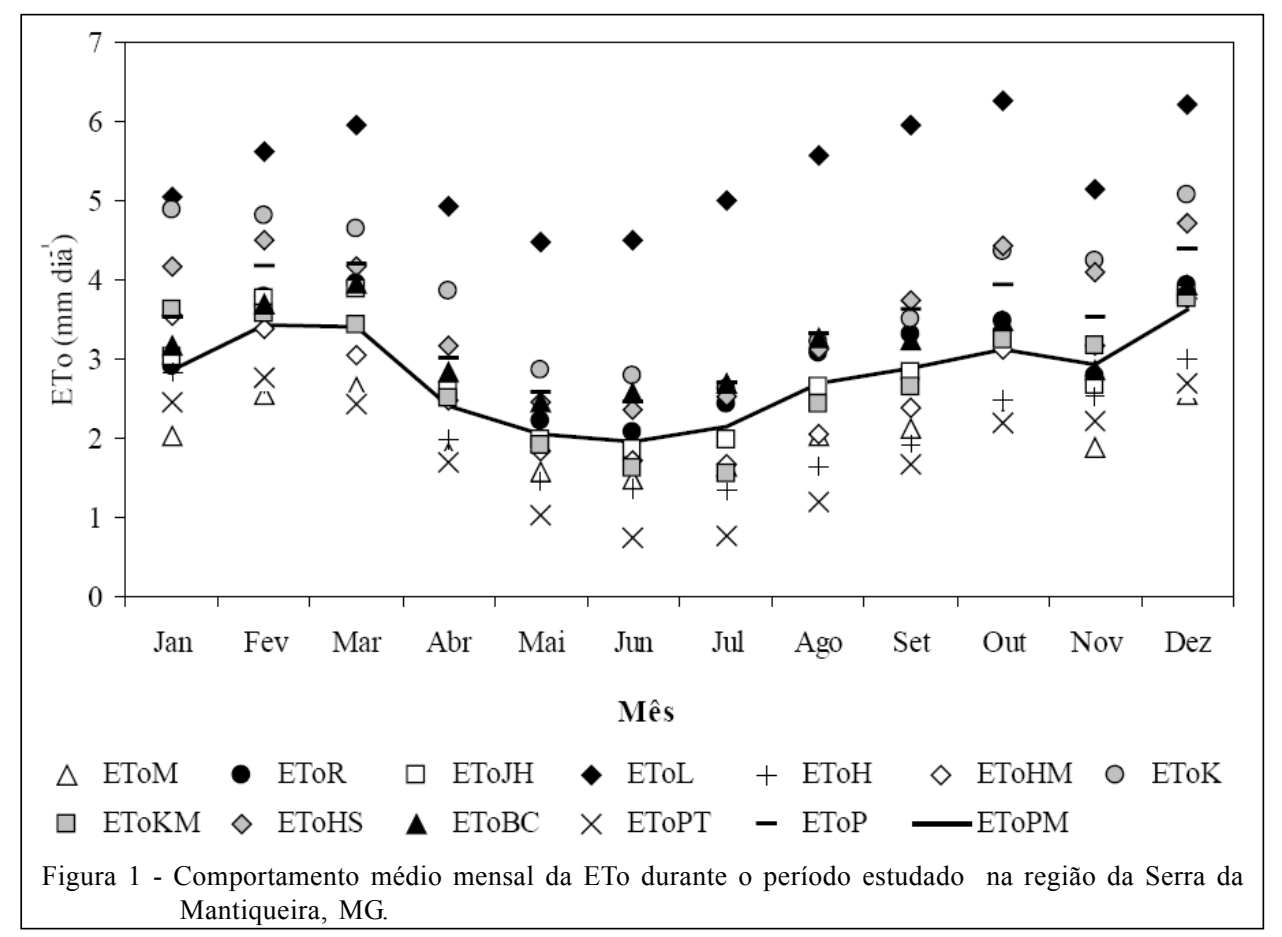

Ciência Rural, v.39, n.9, dez, 2009. 
caracterizando um desempenho "ótimo" $(0,94)$. Por outro lado, o método EToL superestimou com maior magnitude a ETo, comparado ao método padrão ao longo do ano, com EPE de 2,74mm dia ${ }^{-1}$, sendo classificado com desempenho "péssimo" pelo coeficiente c $(0,28)$. Esse comportamento se deve ao fato de que o modelo EToL utiliza apenas a temperatura do ar e a altitude como variável do local, limitando a representatividade das condições climáticas para efeito de estimativa da ETo.

\section{CONCLUSÕES}

Na região da Serra da Mantiqueira, os métodos de estimativa da evapotranspiração de referência que empregam radiação solar apresentam melhor desempenho que os métodos que utilizam apenas a temperatura do ar.

Os métodos de Jensen-Haise, FAO Radiação, Penman e Blaney-Criddle são adequados para estimar a evapotranspiração de referência em escala diária, independentemente da época do ano, na região da Serra da Mantiqueira. Enquanto que, em escala mensal, o método de Jensen-Haise foi o que apresentou melhor ajuste ao método padrão-FAO, seguido dos métodos Karrufa modificado, FAO radiação e Hamon modificado.

\section{AGRADECIMENTOS}

Os autores agradecem ao CNPq, à FAPEMIG (CAG-APQ-00942-08) e à CEMIG/ANEEL (P\&D 176), pelo apoio financeiro e pelas bolsas de estudo e pesquisa aos autores.

\section{REFERÊNCIAS}

ALLEN, R.G. et al. Crop evapotranspiration. Guidelines for computing crop water requirements. Rome: FAO, 1998. 299 p.

ALLEN, R.G.A. et al. Penman for all seasons. Journal of Irrigation and Drainage Engineering, St. Joseph, v.112, n.4, p.348-386, 1986.

ÁVILA, L.F. Comportamento espacial e temporal da umidade do solo numa bacia hidrográfica na Serra da Mantiqueira, Minas Gerais. 2008. 110f. Dissertação (Mestrado em Engenharia Agrícola) - Curso de Pós-graduação em Engenharia Agrícola, Universidade Federal de Lavras, MG.

BORGES, A.C.; MENDIONDO, E..M. Comparação entre equações empíricas para a estimativa da evapotranspiração de referência na Bacia do Rio Jacupiranga-SP. Revista Brasileira de Engenharia Agrícola e Ambiental, Campina Grande, v.11, n.3, p.293-300, 2007. Disponível em: $<\mathrm{http} / /$ www.scielo.br/scielo.php?pid=S1415$43662007000300008 \&$ script $=$ sci arttext\&tlng $=\mathrm{en}>$. Acesso em 25 nov. 2008 . doi: $10.1590 / \mathrm{S} 1 \overline{4} 15-43662007000300008$
CAMARGO, A.P.; CAMARGO, M.B.P. Uma revisão analítica da evapotranspiração potencial. Bragantia, Campinas, v.59, n.2, p.125-137, 2000. Disponível em: <http://www.scielo.br/ scielo.php?pid $=$ S0006 $-\quad 87052000000200002 \&$ script $=$ sci arttext\&tlng=in $>$. Acesso em 25 nov. 2008. doi: 10.1590/ S0006-87052000000200002

CAMARGO, A.P.; SENTELHAS, P.C. Avaliação do desempenho de diferentes métodos de estimativa da evapotranspiração potencial no estado de São Paulo. Revista Brasileira de Agrometeorologia, Santa Maria, v.5, n.1, p.89-97, 1997.

CHAUHAN, S.; SHRIVASTAVA, R.K. Performance evaluation of reference evapotranspiration using climate based methods and artificial neural networks. Water Resources Management, Nova York, v.23, n.5, p.825-837, 2009. Disponível em: <http:/ /www.springerlink.com/content/r4415881p62611q0/>. Acesso em 05 abr. 2009. doi:10.1007/s11269-008-9301-5.

CONCEIÇÃO, M.A.F.; MANDELLI, F. Comparação entre metodos de estimativa da evapotranspiração de referência em Bento Gonçalves, RS. Revista Brasileira de Agrometeorologia, Campinas, v.13, n.2, p. 303-307, 2005.

DOORENBOS, J.; PRUITT, J.O. Crop water requirement. Rome: FAO, 1977. 144p.

MEDEIROS, A.T. et al. Estimativa da evapotranspiração de referência a partir da equação de Penman-Monteith, de medidas lisimétricas e de equações empíricas, em Paraipaba-CE. Revista Engenharia Agrícola, Jaboticabal, v.23, n.1, p.31-40, 2003.

MENDONÇA, J.C. et al. Comparação entre métodos de estimativa da evapotranspiração de referência (ETo) na região Norte Fluminense, RJ. Revista Brasileira de Engenharia Agrícola e Ambiental, Campina Grande, v.7, n.2, p.275-279, 2003. Disponível em: <http:/ /www.scielo.br/scielo.php?script $=$ sci pdf\&pid $=$ S 1415 $43662003000200015 \& \operatorname{lng}=$ en $\& n r m=i s o \& t \operatorname{lng}=p t>$. Acesso em 25 out. 2008.

OLIVEIRA, R.Z. et al. Comparação de metodologias de estimativa da evapotranspiração de referência para a região de Goiânia, GO. Bioscience Journal, Uberlândia, v.21, n.3, p.9-27, 2005 .

PEREIRA, A.R. et al. Evapo(transpi)ração. Piracicaba: FEALQ, 1997. 183p.

REIS, E.F. et al. Estudo comparativo da estimativa da evapotranspiração de referência para três localidades do estado do Espírito Santo no período seco. Idesia, Arica, v..25, n.3, p. $75-84,2007$.

SENTELHAS, P.C. et al. Variação sazonal do parâmetro de Priestley-Taylor para estimativa diária da evapotranspiração de referência. Revista Brasileira de Agrometeorologia, Santa Maria, v.8, n.1, p.49-53, 2000.

VISWANADHAM, Y. et al. The Priestley-Taylor parameter for the Amazon Forest. Forest Ecology and Management, Amsterdam, v. 38, n. 3/4, p. 211-25, 1991.

WILLMOTT, C.J. et al. Statistics for evaluation and comparison of models. Journal of Geophysical Research, Washington, v.0, n.C5, p.8995-9005, 1985.

WILSON, R.G.; ROUSE, W.R. Moisture and temperature limits of the equilibrium evapotranspiration model. Journal of Applied Meteorology, Boston, v.11, n.11, p.436-42, 1972. 